\title{
FACTORS INVOLVED IN SOME CASES OF PLEURAL FLUID ASSOCIATED WITH NORMAL OR INCREASED VOCAL RESONANCE*
}

\author{
CHARLES M. MONTGOMERY, M.D. \\ AND \\ ENGELHARDT A. ECKHARDT, PH.D. \\ PHILADELPHIA
}

Of the many complicated problems in the field of the mechanism of the physical signs of the respiratory system, perhaps none has been so admittedly puzzling, particularly in adults, as that of the normal or increased vocal resonance sometimes encountered in cases of pleural effusion. Skoda thought he had solved the riddle by attributing the phenomenon to what he calls consonance, but his explanation is untenable. The literature on the subject is, for the most part, rather suggestive than really helpful. Walshe's statement is: "Sometimes explicable by solid adhesions conveying the vibrations from the lung to the chest wall; in other instances the anomaly does not admit of explanation."

In trying to obtain any explanation - and only one will be offered here - for the occurrence of normal or increased vocal resonance in cases of pleural effusion, it is necessary first of all to have some knowledge of the factors operating in those cases of effusion in which there occurs a diminution or absence of vocal resonance. Assuming that in most cases of pleural effusion the compressed lung still retains more or less air in the alveoli, an occurrence which is certainly very often encountered, and which we find in experimentally produced effusions in which the conduction of sound is impeded more on the side with the effusion than on the normal side, it is not difficult in these cases, with some air still remaining in the lung, to locate a place where much of the loss of sound might occur, namely, at the junction of the lung with the fluid. Experimentally, it can be readily shown that a marked "break" does occur as sound passes from such an air-bearing lung to fluid. This "break" is apparently largely due to the change of mediums, the air-borne sounds within the lung having to pass from air, which is a very light medium, to fluid which is a very much more dense medium. The loss of sound is not nearly so great as it passes from the fluid to the chest wall. A second factor in diminshing the sounds after they leave the lung is that of diffusion. The intensity of the sounds immediately after entering the fluid as compared to the intensity after

\footnotetext{
* Submitted for publication Dec. 8, 1914.
} 
the sounds reach the chest walls is, in a general way, inversely proportionate to the area of visceral pleura exposed to the fluid in comparison to that of the parietal pleura bathed by the fluid. Recognizing the important part the lung plays in the interference with sound conduction in cases of effusion in which the vocal resonance is diminished or absent, the next logical step in trying to explain those cases exhibiting a normal or increased vocal resonance is to test out other conditions of the lung, to see if any of them might under the circumstances contribute more favorable factors to the conduction of sound.

The condition of the lung offering perhaps the most marked contrast to the air-bearing lung is the completely solidified lung. Having no case to report in which the patient presented before death a normal or increased vocal resonance, and at necropsy a "solid" lung surrounded by fluid, we are obliged to resort to other means for determining whether a "solid" lung would be favorable to the conduction of sound in cases of pleural effusion.

Our most illuminating experiments along this line have been with the chests of fetal calves near term. The alveoli in such animals being free from air, we are enabled to deal with one type of "solid" lung. Sounds introduced into the trachea of such an animal reach the external chest surface with great distinctness. If fluid is introduced into the pleural cavity of a fetal calf till it is completely filled, the sounds at the periphery of the chest will be found to have undergone comparatively little change, being still very distinct, while if the water in the pleural cavity is replaced by air, the sounds at the external chest surface will be found to have undergone a very marked diminution. If the fetal chest contains fluid and the lung is inflated, the sounds at the periphery of the chest will be found to be much weaker than while the lung was still air-free.

An experiment comparable to the foregoing with comparable results can be performed with the fetal lung outside the body. If the fetal lung is submerged in a vessel containing water, and sounds are introduced into the trachea and if a stethoscope is used, the mouth of the bell being tightly covered with a piece of liver to represent chest wall, and if this liver is likewise placed in contact with the water, but at no point touching the lung, the sounds will be heard with great distinctness after having passed through fetal lung, water and liver. Very much weaker sounds will be obtained if the fetal lung is replaced by an air-bearing lung.

Another experiment closely resembling the preceding ones can be made by replacing, in the last experiment, the fetal lung by a heart. Sounds after entering the aorta and passing through the cardiac walls, 
the fluid and the liver covering the inferior opening of the stethoscope, still retain considerable intensity.

One can readily become convinced of the facility with which sounds pass from "solid" tissue to water by applying a suitable cylinder to the chest wall of a living human being and inserting water into this cylinder so that it is in contact with the chest wall, while the opposite end of the cylinder is tightly joined to the bell of the stethoscope. The vocal resonance and the respiratory murmur, though diminished, will be very distinctly audible.

How closely do our experiments parallel conditions that may occur in the human subject? In each experiment except the last, as contrasted with the human being, there are three things to be compared: the chest wall (or its representative, liver), the fluid and the lung. The fetal calf's chest wall corresponds sufficiently closely to the adult chest wall. Even if it is, so to spreak, made thicker by overlaying it with liver, much of the sound intensity is retained. As for the fluid, conditions are relatively much alike in both the experiments and in the supposed clinical case. The amount of diffusion by the fluid may be alike in both cases. Any striking difference, then, in the acoustic phenomena in the experiments and in human beings must relate to the lungs themselves.

The great bulk of the soft cellular tissues, whether normal, inflammatory, or malignant, apart from their fluid contents, is composed of substances known as gels. These gels, of which gelatin in a 10 or 20 per cent. strength in the firm state is an example in pure form, are closely allied in their general physical characteristics, and one would expect this similarity to extend to their acoustic properties. This has been just what has occurred in all our experimental work. We have had sufficient material for experimentation in the shape of fetal lung, liver, and muscle, and though more restricted in obtaining "solid" material from hepatized lungs, experimentation and inference show that such diseased tissue in its sound-conducting properties closely resembles the soft tissues of the body, including the fetal lung.

All our evidence indicates that these various substances, liver, muscle, fetal lung, and hepatized lung greatly resemble one another in the following particulars: They are all fairly good conductors of sound. In a general way they considerably resemble one another in their conductivity of sound. When one or more of these materials are placed in apposition the resultant effect on sound conduction is much the same as if a similar amount of any single one of these materials had been used, indicating that no serious "break" occurs as sound passes from one of these substances to another. Finally, in their relation to air and water they all resemble one another closely; that is, 
there is a marked loss of sound as it passes from air to any of these substances, while there is very much less loss of sound as it passes from these various substances to air, and the passage of sound from these substances to water or in the reverse direction is attended with no serious loss of sound due to the change of mediums, because they are not of sufficiently different densities. We conclude, therefore, that these various materials, liver, muscle, fetal lung, and hepatized lung, may be considered for our purposes to be one single medium, and to act practically alike in their relation to sound conduction, whether by themselves or in association with other mediums.

While our chain of evidence is not absolutely complete as far as the lung affected with solid infiltration is concerned, all available evidence indicates that it corresponds acoustically to fetal lung and other gels.

The fluid contents of these various substances does not materially affect their conductivity.

When a pleural effusion exists there may be associated either one of two types of "solid" lung; first, that in which there is no infiltrative process, but from which the air has disappeared as the result of pressure or absorption, leaving a lung closely resembling the fetal type; and, secondly, the lung which is "solid" as the result of inflammatory or malignant change. The airless but otherwise normal lung would, on account of its smaller bulk, probably offer more favorable opportunities for sound conduction than a hepatized lung, provided that in the two cases the bronchi were equally patulous.

It is possible, according to Skoda, for only a portion of a lung to become airless as a result of an effusion, and to sink beneath the surface of the fluid.

If our conclusion is correct, that the tissue of a lung "solid" either from compression or actual pulmonary disease closely resembles fetal lung tissue acoustically, it is clear how the vocal resonance might be normal or increased in cases of pleural effusion. An increase in the vocal resonance in cases of consolidation of the lung is the usual clinical finding, and as long as the bronchi remain patulous the mere presence of fluid between the "solid" lung and the chest wall should not very materially affect the transmission of sounds, except through diffusion, though the change of medium from "solid" to fluid, and from fluid to "solid," would exert more or less of an effect. The reasons that no great change except that due to diffusion should take place are, first, that water itself is a good conductor of sound, and, secondly, that sound vibrations do not suffer greatly in intensity as they pass from "solid" tissue to water or from water to "solid" tissue. This latter fact might be expected to occur, because water and "solid" tissue do not differ greatly in density; but the same thing can be readily demon- 
strated by a great variety of experiments, such as those of the fetal calf and the others presented above.

Our conception, then, of what we believe occurs in some cases of pleural effusion with a normal or increased vocal resonance may be summarized as follows: The sounds reach the surface of the "solid" lung with a certain degree of intensity, which is naturally greater than would be encountered at the external chest surface in a case of pneumonia without effusion, because the sound has not yet passed through the chest wall. The intensity of sound at the lung surface will vary to some extent with the amount of lung tissue that is traversed, so that we would expect to find louder sounds at the surface of a lung that is airless as a result of compression than in one that is "solid" from infiltration. The vibrations then enter the fluid, and do this without any serious "break" as a result of the change of medium. In their course through the fluid comparatively little sound is lost except that due to diffusion, because of the excellent conducting properties of fluid. Finally, the vibrations enter the chest wall, and again effect a transfer of medium without great loss of intensity. In other words, the interference in sound conduction is not very greatly different, as a result of the fluid in the pleural cavity, from a case presenting a "solid" lung without any effusion, provided the bronchi remain patulous, except for the loss due to diffusion. It is certainly true that the conditions for sound transmission are much better in a case with a "solid" lung surrounded by fluid than in a case of effusion in which the lung still contains air, or in a case of pneumothorax, whether the lung is either "solid" or air-bearing, provided there is no perforation from the lung into the pleural cavity.

If our explanation is correct, why does not this phenomenon occur oftener? As a matter of fact, it is impossible to state just how often a normal or increased vocal resonance does occur in cases of pleural effusion. Small basal accumulations of fluid overlying "solid" lung giving a normal or increased vocal resonance, are probably not very rare. As to the actual causes for the infrequency of the phenomenon, they may be considered to lie chiefly in the lung substance or in the bronchi. We have already spoken of the marked loss of sound that occurs when a lung, still retaining air in the parenchyma, is in contact with fluid. Interference with sound transmission from bronchial obstruction might, theoretically, be caused either by pressure from without, thus bringing the bronchial walls in apposition, or by edema or other abnormal fluid within the bronchi, which, through the action of fluid, or fluid and air, that is, froth, would occasion an intrabronchial obstruction to the passage of sounds. 
Our conclusions are offered in explanation of only some of the cases of pleural effusion manifesting a normal or increased vocal resonance, for we do not believe the explanation holds good in all cases, and especially in children we believe that other causes may operate.

The loss in intensity due to diffusion in both the air-bearing and "solid" lungs will be rendered less by whatever allows the lung to approach closer to the chest wall.

The conclusions apply to various forms of fluid in the pleural cavity, whether serous, purulent or hemorrhagic (fluid blood).

The experimental evidence on which the conclusions are based has heen reduced to a minimum amount of space, but we trust that it is sufficient to elucidate the subject in hand. The experiments in full will be given elsewhere.

This subject has clinical as well as theoretical interest. Thus, in any case of a doubtful nature presenting a normal or increased vocal resonance, the idea of fluid in the pleural cavity should be entertained; and, given a case with demonstrable fluid in the pleural cavity exhibiting clinically a normal or increased vocal resonance, the possibility of there being "solid" lung beyond the fluid should be borne in mind. While in some cases such a "solid" lung may be simply a compressed and airless lung, the other alternative is also to be considered, that the lung may be affected with inflammatory or malignant disease. If the condition is a recent one, recently developed consolidation of the lung in addition to the fluid is to be suspected.

2210 Locust Street-5647 Kingsessing Avenue. 